\title{
Analysis of the combination of the slider-crank and cam mechanism to drive the automatic bandsaw blade grinder
}

\author{
Tan Dang Nguyen*, Thang Hong Thi Le, Giap Van Doan
}

Hanoi University of Mining and Geology, Hanoi, Vietnam

\begin{abstract}
ARTICLE INFO
ABSTRACT

Article history:

Received 02nd Apr. 2021

Revised 05th July 2021

Accepted 01 ${ }^{\text {st }}$ Aug. 2021

Keywords:

Analytic method,

Cam mechanism,

Combination,

Slide crank mechanism.

A slide crank and cam mechanism can be combined and used to drive a bandsaw blade grinder. The movement of these mechanisms must ensure a harmonious combination to avoid the impacts of a collision and dynamic load that appears on the cam and roller surfaces. The technical parameters can be determined by methods of geometry, or 3D simulation, or analytical methods. In order to flexibly design the cam and slide crank mechanism, this study uses the analytic method. By setting up mathematical formulas and applying Mathcad software, it allows to quickly determine and change the initial parameters of the mechanisms, evaluates their changes to the stroke and structural profile. Simultaneously, the graphs of the stroke, velocity, and acceleration of the cam mechanism allow the designer to evaluate and select suitable parameters.
\end{abstract}

Copyright (C) 2021 Hanoi University of Mining and Geology. All rights reserved.

${ }^{*}$ Corresponding author

E - mail: nguyendangtan@humg.edu.vn

DOI: 10.46326/JMES.2021.62(4).10 


\title{
Xây dựng biên dạng cam tịnh tiến và hành trình cơ cấu tay quay con trượt dùng truyền động cho máy mài lưỡi cưa vòng tự động
}

\author{
Nguyễn Đăng Tấn, Lê Thị Hồng Thắng, Đoàn Văn Giáp
}

Trường Đại học Mỏ - Địa chất, Hà Nội, Việt Nam

\begin{abstract}
THÔNG TIN BÀI BÁO
TÓM TẮT

Quá trình:

Nhận bài 02/4/2021

Sửa xong $05 / 7 / 2021$

Chap nhận đăng 01/8/2021

\section{Tù̀ khóa:}

Cơ cấu cam,

Kết hợp các cơ cấu, Phương pháp giải tích, Tay quay con trượt.

Cơ cấu cam kết hợp với tay quay con trượt được sủ dung để truyền động cho cơ cấu đẩy lưỡi cưa và di chuyển đá mài máy mài lưỡi cưa vòng tự động. Chuyển động của cơ cấu này phải đảm bảo kết hợp hài hòa với nhau để tránh va đập cũng như tải trọng động va đập xuất hiện trên bề mặt cam và con lăn. Để xác định các thông số của cơ cấu, có thể sư dụng phương pháp hình học, mô phỏng mô hình $3 D$ hoặc phương pháp giải tích. Phương pháp hình học tiến hành thủ công, tốn nhiều thời gian thiết kế. Phương pháp xây dưng mô hình mô phỏng 3D thường áp dụng khi có kích thước của cơ cấu hoặc kích thước sơ bộ. Khi thay đổi các thông số hình học của cơ cấu cần phải xây dựng lại mô hình mói. Do đó tính linh hoạt chưa cao và tốn nhiều thò̀i gian xây dựng mô hình. Để thiết kế cơ cấu cam kết hợp với tay quay con trượt một cách linh hoạt khi thay đổi các thông số đầu vào, bài báo sư dụng phương pháp giải tích. Bằng việc thiết lập các công thức toán hoc và úng dung phần mềm Mathcad cho phép xác định nhanh chóng thay đổi các thông số ban đầu của bài toán, đánh giá được thay đổi của chúng đến hành trình, biên dạng cơ cấu. Đồng thòi, các đồ thị biểu diễn hành trình, vận tốc, gia tốc cơ cấu cam cho phép thiết kế đánh giá, lựa chọn các thông số phù hợp. Kết quả nghiên cúu cho phép xác định biên dạng cam tịnh tiến cho máy mài.
\end{abstract}

C 2021 Trường Đại học Mỏ - Địa chất. Tất cả các quyền được bảo đảm.

\section{Mở đầu}

Lưỡi cưa vòng là loại lưỡi cưa dài, sắc nhọn bao gồm nhiều răng cưa bằng hợp kim hoặc thép, được lắp vào các máy cưa vòng để cắt vật liệu. Do có độ dài khá lớn cùng với răng cưa sắc nhọn nên lưỡi cưa vòng có thể cắt được đa dạng các loại vật liệu như thép hình, thép ống, gỗ, đá,... Tùy theo vật liệu được cắt mà có thể lựa chọn lưỡi cưa với bước răng khác nhau nhằm mục đích tạo ra năng suất và chất lượng bề mặt cắt phù hợp. Lưỡi cưa máy xẻ gỗ có các thông số cơ bản như chiều dày, rộng, dài cũng như bước răng, chiều sâu răng (Hình 1).

Sau một thời gian sử dụng, lưỡi cưa bị mòn ở đỉnh răng và các cạnh của răng bị tròn đầu. Để có thể tiếp tục sử dụng, người dùng cần phải mài lưỡi

\footnotetext{
*Tác giả liên hệ

E - mail: nguyendangtan@humg.edu.vn

DOI: 10.46326/JMES.2021.62(4).10
} 

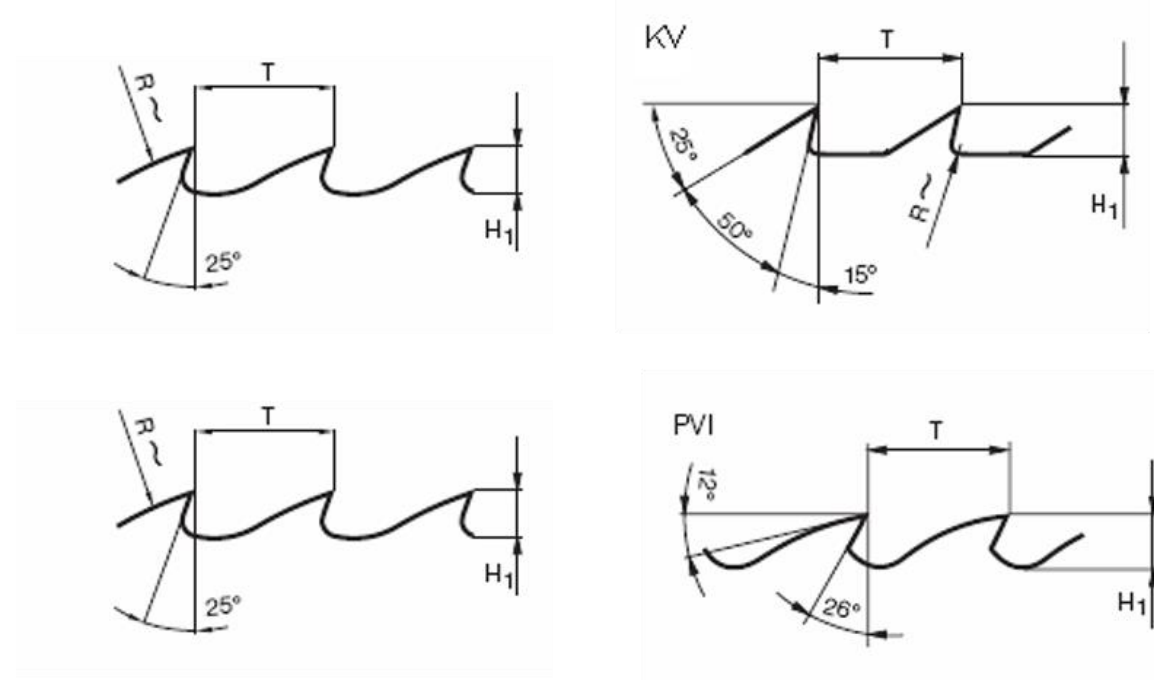

Hình 1. Hình dáng hình học một vài loại lưỡi cưa (A\&B Tool).

cưa. Hiện nay, nhiều loại máy mài lưỡi cưa được nghiên cứu, chế tạo nhằm tăng năng suất cũng như chất lượng mài lưỡi cưa. Quá trình mài lưỡi cưa trên máy cần hai chuyển động cơ bản: chuyển động ra vào của đá mài và chuyển động đẩy lưỡi cưa sang răng kế tiếp (Hình 2). Trong đó: T - bước

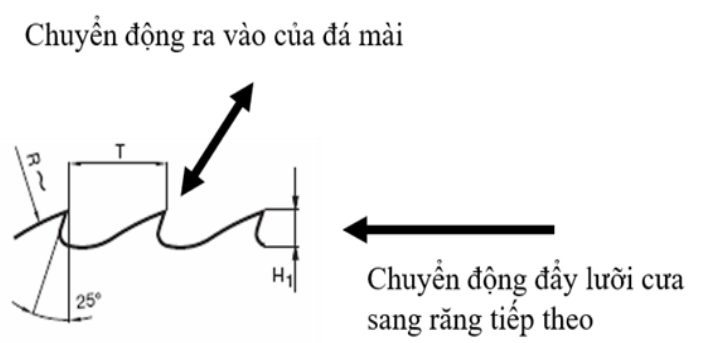

Hình 2. Chuyển động cần thiết của cơ cấu dẫn động.

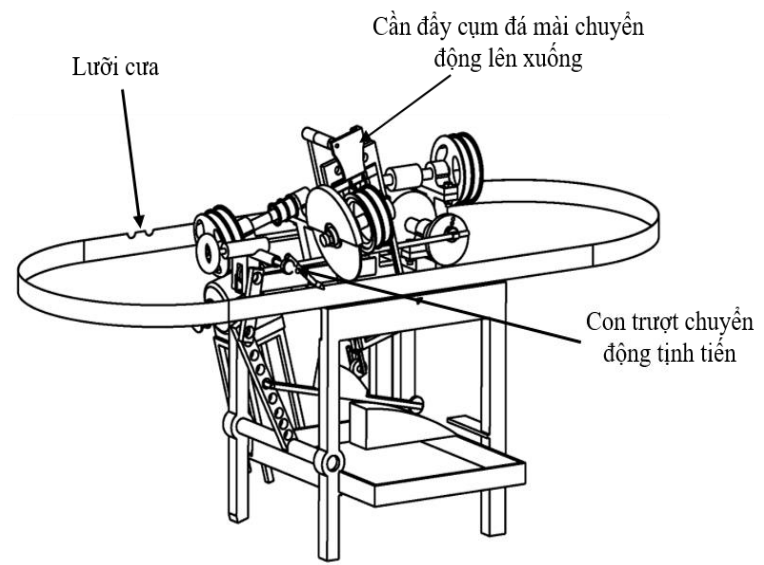

Hình 3. Cấu tạo máy mài lưỡi cưa vòng. răng (mm), R - bán kính cong răng cưa (mm), $\mathrm{H}_{1}$ chiều cao từ phần đỉnh đến phần bụng lưỡi cưa (mm).

Có nhiều loại máy mài lưỡi cưa với cấu tạo khác nhau. Về cơ bản, một máy mài lưỡi cưa bao gồm: cơ cấu đẩy đá mài và cơ cấu đẩy lưỡi cưa sang bước tiếp theo sau khi kết thúc mài một răng cưa (Hình 3). Lưỡi cưa có thể đặt nằm ngang hoặc thẳng đứng.

Trong quá trình mài, đá mài nghiêng một góc bằng góc nghiêng của răng cũng như cơ cấu đẩy lưỡi cưa phải đẩy hành trình bằng bước răng T. Để đảm bảo không có va đập giữa hai chuyển động này, có thể sử dụng hai cơ cấu dẫn động riêng và điều khiển làm việc kết hợp với nhau. Biện pháp này có độ linh hoạt cao, tuy nhiên chi phí điều khiển lớn cũng như hệ điều khiển phức tạp. Để khắc phục vấn đề dẫn động không đồng bộ, hai cơ cấu đẩy lưỡi cưa và đá mài được dẫn động chung (Nhật Phương, 2021). Nghĩa là, một động cơ dẫn động cho hai cơ cấu và liên kết với nhau bằng các khâu, khớp cơ khí.

Để thực hiện các chuyển động này, có thể sử dụng các cơ cấu cơ khí như: bốn khâu bản lề, bánh răng, truyền động đai, truyền động xích,... được sử dụng khá phổ biến trên thế giới với nhiều lĩnh vực khác nhau (Trịnh Chất, Lê Văn Uyển, 2000). Nhờ vào cơ cấu cam, có thể thiết kế tạo ra pha và hành trình chuyển động của khâu bị dẫn theo yêu cầu mà những cơ cấu cơ khí khác không thực hiện được. Bằng việc phối hợp các chuyển động, cơ cấu cam được dùng để dẫn động các chuyển động lắc 
hoặc tịnh tiến của cần cam cho các máy từ đơn giản đến phức tạp. Cơ cấu bốn khâu bản lề được dùng biến chuyển động quay thành chuyển động lắc, dạng biến thể của nó là cơ cấu tay quay con trượt (Đinh Gia Tường, Tạ Khánh Lâm, 2003). Việc nghiên cứu các cơ cấu cam cũng như cơ cấu tay quay con trượt riêng lẻ được chỉ ra trong các tài liệu về nguyên lý máy (Trần Quang Minh, Nguyễn Quang Hưng, 2015).

Đối với cơ cấu tay quay con trượt, các thông số hình học như chiều dài tay quay và thanh truyền, độ lệch tâm cũng như vị trí góc của tay quay sẽ xác định vị trí của con trượt. Khi các góc quay biến đổi theo thời gian, hành trình chuyển động của con trượt sẽ phụ thuộc vào góc quay này (Luck K., Modler K-H, 1990). Đồ thị quay hệ giữa góc quay và hành trình chuyển động đóng vai trò quyết định cho việc kết hợp cơ cấu này với các cơ cấu khác của máy (Jack Holman, John Loyd, 1999). Để có thể điều chỉnh được hành trình chuyển động của con trượt, tay quay có thể làm dạng đĩa, trên đó có để các vị trí bán kính khác nhau để lắp ghép với thanh truyền. Khi muốn thay đổi hành trình của con trượt, có thể điều chỉnh bán kính tay quay (Nguyễn Trọng Hiệp, 1997).

Cơ cấu cam được từng bước hoàn thiện để có thể đáp ứng được những yêu cầu khắt khe trong truyền động (Corvers B., Hüsing M., 2015; Andreas F. và nnk., 2015). Ngoài ra, với sự hỗ trợ bằng các phần mềm thiết kế CAD-3D, chuyển động của các cơ cấu dẫn động bằng cam cùng các thông số động học, động lực học được tính toán, mô phỏng. Sau đó, biên dạng cơ cấu cam được xuất ra file dữ liệu Numerical Control (NC) để gia công trên các máy CNC (Maik Berger, 2011).

Nghiên cứu về quy luật chuyển động của cơ cấu tay quay con trượt cũng như cơ cấu cam đã được thể hiện ở nhiều công trình nghiên cứu đã được đề cập ở trên. Tuy nhiên, khi tính toán thiết kế máy, người thiết kế có thể sử dụng cơ sở lý thuyết này để vận dụng cho bài toán cụ thể.

Khi tay quay của cơ cấu tay quay con trượt quay với góc quay $\varphi$, con trượt sẽ chuyển động tịnh tiến. Khác với cơ cấu cam, khi tay quay chuyển động quay tròn, thông qua thanh truyền làm con trượt chuyển động tịnh tiến. Theo quan hệ hình học, con trượt luôn chuyển động mà không có hành trình nghỉ như cơ cấu cam. Để có thể kết hợp hai cơ cấu này nhằm dẫn động cho máy mài lưỡi cưa, cần xác lập pha làm việc của chúng theo góc quay $\varphi$. Theo góc quay cơ cấu dẫn động, khi đá mài chuyển động xuống để mài lưỡi cưa, lưỡi cưa phải được giữ chặt, lúc này con trượt đẩy lưỡi cưa ở hành trình về. Khi con trượt đẩy lưới cưa tiến lên một răng sau quá trình mài một răng kết thúc, cơ cấu dẫn động đá mài phải đang đi lên. Thời điểm đẩy lưỡi cưa, đá mài phải ở phía bên ngoài lưỡi cưa. Do vậy, khi thiết kế cụm truyền động, yêu cầu các chuyển động của con trượt và cần đẩy nghịch hướng nhau.

Bằng phương pháp thiết kế truyền thống hay mô phỏng, người thiết kế đều phải xây dựng được pha chuyển động cũng như biên dạng bề mặt cơ cấu cam. Để tiết kiệm thời gian thiết kế cũng như linh hoạt trong thay đổi kích thước ban đầu của hệ truyền động, bài báo trình bày phương pháp thiết kế biên dạng cam và xây dựng đồ thị pha truyền động bằng phương pháp giải tích. Phần mềm Mathcad cho phép xác định nhanh chóng các thông số đầu ra khi thay đổi thông số đầu vào cũng như hiển thị pha chuyển động của chúng.

\section{Nội dung nghiên cứu}

\subsection{Xây dựng thông số động học cơ cấu}

Trong thông số dẫn động và bị động, độ lớn động học được xác định như hàm chuyển đổi chuyển động của khâu chủ động và khâu bị động. Thông số chuyển động của khâu chủ động được xác định bằng biến số $\mathrm{x}$, nó đại diện cho góc quay hoặc quãng đường chuyển động. Thông số chuyển động của khâu bị động được xác định tương ứng là góc quay hoặc hành trình chuyển động và được đại diện bằng biến số $\mathrm{y}$ (Johannes Volmer, 1989).

Hiện tại, có hai loại cơ cấu cam được sử dụng phổ biến là cơ cấu cam cần lắc hoặc cơ cấu cam cần tịnh tiến. Để đẩy đá mài chuyển động lên xuống, mô hình cam có cần chuyển động tịnh tiến được lựa chọn, sơ đồ cơ cấu được chỉ ra trên Hình 4 . Trong mô hình này, cam chuyển động tịnh tiến nhờ cơ cấu tay quay con trượt, trên bề mặt cam có liên kết cần tịnh tiến. Khi cam chuyển động tịnh tiến, cần đẩy chuyển động tịnh tiến lên xuống. Để xác định biên dạng hình học bề mặt cam bằng phương pháp giải tích, các biến số và sơ đồ xác định được cho trong Hình 4 bên phải.

Trong đó: $\mathrm{x}$ - hành trình chuyển động của con trượt $(\mathrm{mm}), \mathrm{y}$ - hành trình chuyển động của cam đẩy B (mm). 

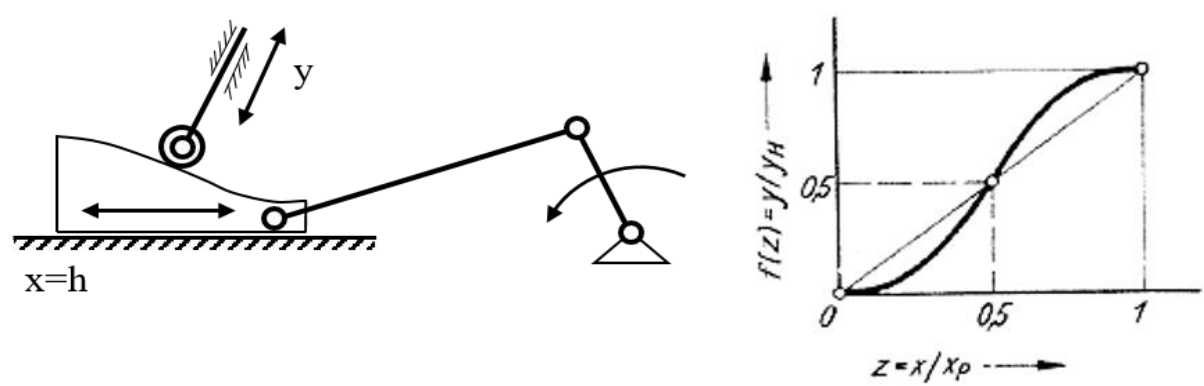

Hình 4. Sơ đồ cam tịnh tiến và xây dựng biên dạng cam.

Gọi $y_{H}$ hành trình lớn nhất của cần đẩy (mm), $x_{P}$ khoảng chuyển động lớn nhất theo chiều $\mathrm{x}$ (mm), hàm số y được xác định như sau:

$$
y=y_{H} f\left(\frac{x}{x_{P}}\right)=y_{H} f(z)
$$

Để chuyển động cần đẩy êm dịu, có nhiều cách lựa chọn biên dạng cam như dạng hình sin, parabol hay đa thức bậc cao. Đa thức bậc cao có ưu điểm biên dạng mượt, đơn giản tính toán các hệ số đa thức cũng như đạo hàm các cấp. Do đó, bài báo lựa chọn bề mặt cam được chọn theo đa thức bậc n như sau:

$$
f(z)=\sum_{i=1}^{n} A_{i} z^{i}
$$

Trong đó: $A_{i}$ - hạng tử thứ i của đa thức, $\mathrm{n}$ bậc của đa thức, $\mathrm{z}$ là biến số được xác định bằng $\frac{x}{x_{P}}$.

Với số mũ càng lớn, đường cong vận tốc, gia tốc êm dịu, không có bước nhảy đột ngột. Tuy nhiên, số mũ lớn làm bài toán xác định các hệ số của đa thức phức tạp hơn. Hiện nay, thường sử dụng các đa thức bậc 4, 5, 6 hoặc 7 .

Với cơ cấu tay quay con trượt, các thông số hình học cơ bản bao gồm: chiều dài các đoạn $l_{2}, l_{3}$ $(\mathrm{mm})$, vị trí khớp liên kết tay quay với thanh truyền $A$, vị trí khớp liên kết giữa thanh truyền và con trượt $B$, khoảng lệch tâm e, góc tạo bởi giữa thanh truyền liên kết với con trượt và đường thẳng nằm ngang $\varphi_{31}\left(^{\circ}\right)$ cũng như góc gốc tọa độ dẫn động $\varphi_{12}(t)\left(^{\circ}\right)$. Để tính toán thiết kế cơ cấu tay quay con trượt, cần phải xác định hành trình $s=s\left(\varphi_{21}\right)(\mathrm{mm})$, vận tốc $\dot{s}=v_{B}(\mathrm{~m} / \mathrm{s})$, gia tốc $\ddot{s}=a_{B}\left(\mathrm{~m} / \mathrm{s}^{2}\right)$. Các thông số hình học được cho trên Hình 5 .

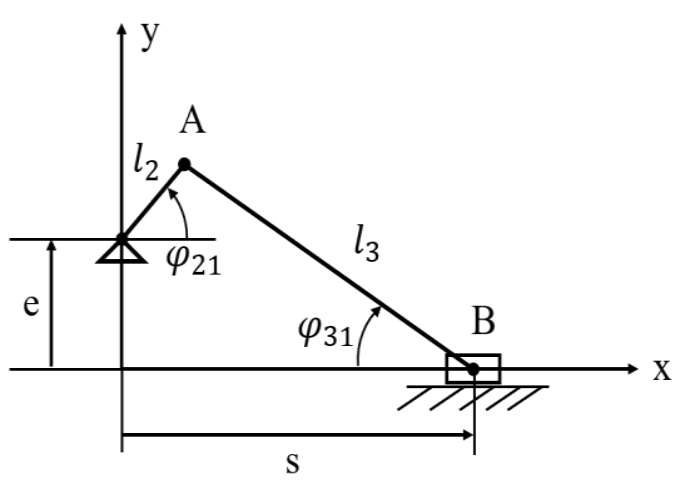

Hình 5. Sơ đồ tính toán tay quay con trượt.

Để xác định mối quan hệ giữa quãng đường chuyển động s (mm) theo hệ trục tọa độ $0 x y$, chiếu các chuyển động lên trục đứng và trục ngang, ta có:

$$
\begin{aligned}
& l_{2} \cos \varphi_{21}+l_{3} \cos \varphi_{31}-s=0 \\
& l_{2} \sin \varphi_{21}+l_{3} \sin \varphi_{31}+e=0
\end{aligned}
$$

Từ đó xác định được

$$
\begin{gathered}
\sin \varphi_{31}=-\frac{e+l_{2} \sin \varphi_{21}}{l_{3}} \\
\cos \varphi_{31}=\sqrt{1-\left(\frac{e+l_{2} \sin \varphi_{21}}{l_{3}}\right)^{2}}
\end{gathered}
$$

Khi góc $\varphi_{31}$ được xác định, hành trình $\mathrm{s}$ của con trượt phụ thuộc góc tay quay $\varphi_{21}$ được xác định như sau:

$$
s=l_{2} \cos \varphi_{21}+\sqrt{l_{3}^{2}-\left(e+l_{2} \sin \varphi_{21}\right)^{2}}
$$

Vận tốc góc khâu 3 theo thời gian quy về khâu dẫn được xác định:

$$
\varphi_{31}^{\cdot}=-\frac{l_{2} \cos \varphi_{21}}{l_{3} \cos \varphi_{31}} \varphi_{21}^{\cdot}=\varphi_{31}^{\prime} \varphi_{21}^{\cdot}
$$


Vận tốc của con trượt được xác định:

$$
\begin{aligned}
v_{B} & =\dot{s}=l_{2}\left(-\sin \varphi_{21}+\frac{\sin \varphi_{31} \cos \varphi_{31}}{\cos \varphi_{31}}\right) \varphi_{21} \\
& =s^{\prime} \varphi_{21}
\end{aligned}
$$

Hệ phương trình để xác định gia tốc bằng cách đạo hàm theo thời gian vận tốc:

$$
\begin{aligned}
& -l_{2} \sin \varphi_{31} \ddot{\varphi_{31}}-\ddot{s} \\
& =l_{2} \sin \varphi_{21} \ddot{\varphi_{21}} \\
& +l_{2} \cos \varphi_{21} \varphi_{21}{ }^{2} \\
& +l_{3} \cos \varphi_{31} \dot{\varphi}_{31}^{2} \\
& l_{3} \cos \varphi_{31} \ddot{\varphi_{31}}=-l_{2} \cos \varphi_{21} \ddot{\varphi_{21}} \\
& +l_{2} \sin \varphi_{21} \dot{\varphi}_{21}{ }^{2} \\
& +l_{3} \sin \varphi_{31} \dot{\varphi}_{31}^{2}
\end{aligned}
$$

Đặt tỷ lệ giữa chiều dài tay quay và chiều dài thanh truyền $\lambda=\frac{l_{2}}{l_{3}}$. Nghiên cứu này, chọn độ lệch tâm e $=0$. Các thành phần góc quay, vận tốc, gia tốc của thanh truyền được xác định:

$$
\begin{gathered}
\varphi_{31}=-\arcsin \left(\lambda \sin \varphi_{21}\right) \\
\dot{\varphi}_{31}^{\cdot}=\frac{-\lambda \cos \varphi_{21}}{\sqrt{1-\lambda^{2} \sin ^{2} \varphi_{21}}} \quad=\varphi_{31}{ }^{\prime} \varphi_{21} \\
\ddot{\varphi_{31}} \\
=\frac{-\lambda \cos \varphi_{21}}{\sqrt{1-\lambda^{2} \sin ^{2} \varphi_{21}}} \varphi_{21}^{.} \\
+\frac{\lambda \sin _{21}}{\sqrt{1-\lambda^{2} \sin ^{2} \varphi_{21}}}(1 \\
\left.-\frac{\lambda^{2} \cos ^{2} \varphi_{21}}{1-\lambda^{2} \sin ^{2} \varphi_{21}}\right) \varphi_{21}^{2}
\end{gathered}
$$

Hành trình chuyển động:

$$
s=l_{2} \cos \varphi_{21}+l_{3} \sqrt{1-\lambda^{2} \sin ^{2} \varphi_{21}}
$$

Vận tốc:

$$
v_{B}=-l_{2} \sin \varphi_{21}\left(1+\frac{\lambda \cos \varphi_{21}}{\sqrt{1-\lambda^{2} \sin ^{2} \varphi_{21}}}\right) \varphi_{21}^{\cdot}
$$

Gia tốc:

$$
\begin{aligned}
& a_{B}= \\
& -l_{2}\left[\begin{array}{c}
\sin \varphi\left(1+\frac{\lambda \cos \varphi_{21}}{\sqrt{1-\lambda^{2} \sin ^{2} \varphi_{21}}}\right) \ddot{\varphi_{21}}+ \\
\left(\cos \varphi_{21}-\frac{\lambda\left(1-2 \cos ^{2} \varphi_{21}-\frac{\lambda^{3} \sin ^{2} \varphi_{21} \cos ^{2} \varphi_{21}}{1-\lambda^{3} \sin ^{2} \varphi_{31}}\right)}{\sqrt{1-\lambda^{2} \sin ^{2} \varphi_{21}}}\right) \varphi_{21}^{2}
\end{array}\right]
\end{aligned}
$$

Trong thực tế, để đảm bảo tay quay chuyển động quay tròn, hệ số tỷ lệ $\lambda$ được chọn nhỏ hơn 1. Do đó, các thành phần vận tốc, gia tốc quay, vận tốc, gia tốc chuyển động tịnh tiến được xác định như sau:

$$
\begin{gathered}
\varphi_{31} \approx-\lambda \sin \varphi_{21}-\frac{1}{6} \lambda^{3} \sin ^{3} \varphi_{21} \\
\sin \varphi_{31} \approx-\lambda \sin \varphi_{21} \\
\cos \varphi_{31} \approx 1-\frac{1}{2} \lambda^{2} \sin ^{2} \varphi_{21} \\
\varphi_{31}^{\prime}=\approx-\lambda \cos \varphi_{21}-\frac{1}{2} \lambda^{3} \sin ^{2} \varphi_{21} \cos \varphi_{21} \\
\varphi_{31}^{\prime \prime} \approx \lambda \sin \varphi_{21}+\lambda^{3} \sin \varphi_{21}(-1 \\
\left.+\frac{3}{2} \sin ^{2} \varphi_{21}\right) \\
s \approx l_{3}+l_{2}\left(\cos \varphi_{21}\right. \\
-\frac{1}{2} \lambda \sin ^{2} \varphi_{21} \\
\left.-\frac{1}{8} \lambda^{3} \sin ^{4} \varphi_{21}\right)
\end{gathered}
$$

\subsection{Xác định biên dạng cam}

Trong nghiên cứu này, biên dạng cam lựa chọn được xác định bằng đa thức bậc 5 vì đảm bảo mức độ chuyển tiếp biên dạng cam và quá trình tính toán các hệ số của đa thức không quá phức tạp (Johannes Volmer, 1989). Hàm tổng quát đa thức được thể hiện như sau:

$$
\begin{aligned}
f(z)=A_{0}+A_{1} z+A_{2} z^{2}+A_{3} z^{3}+A_{4} z^{4}+ \\
A_{5} z^{5}
\end{aligned}
$$

Tại vị trí ban đầu $\mathrm{z}=0$, đạo hàm đa thức trên để tìm các giá trị, kết quả thu được $A_{0}=0$

Tại vị trí $\mathrm{z}=1, \mathrm{f}(1)=1$ (Hình 4 ). Để đảm bảo không có bước nhảy, yêu cầu $f^{\prime \prime}(0)=0 ; f^{\prime \prime}(1)=$ 0 ; từ đó xác định được $A_{1}=A_{2}=0$

Các thành phần còn lại được xác định như sau:

$$
\begin{aligned}
A_{3} & =10-6 f^{\prime}(0)-4 f^{\prime}(1)-\frac{3}{2} f^{\prime \prime}(0)+ \\
\frac{1}{2} f^{\prime \prime}(1) & =10 \\
A_{4} & =-15+8 f^{\prime}(0)+7 f^{\prime}(1)+\frac{3}{2} f^{\prime \prime}(0)- \\
f^{\prime \prime}(1) & =-15 \\
A_{5} & =6-3\left[f^{\prime}(0)+f^{\prime}(1)\right]-\frac{1}{2}\left[f^{\prime \prime}(0)-\right. \\
\left.f^{\prime \prime}(1)\right] & =6
\end{aligned}
$$


Khi đó hàm đa thức được viết lại như sau:

$$
f(z)=10 z^{3}-15 z^{4}+6 z^{5}
$$

Hành trình chuyển động của con trượt $x=h$ $(\mathrm{mm})$, hành trình cần đẩy $y_{H}(\mathrm{~mm})$ :

$$
\begin{gathered}
h=l_{2} \cos \varphi_{21} \\
h=0, y=y_{H} \\
h=h_{\text {max }}=l_{2}, y=0
\end{gathered}
$$

Trong đó:

$$
\begin{aligned}
y=y_{H} f\left(\frac{h}{l_{2}}\right)= & y_{H}[1 \\
& -\left(10\left(\frac{h}{l_{2}}\right)^{3}-15\left(\frac{h}{2 l_{2}}\right)^{4}\right. \\
& \left.\left.+6\left(\frac{h}{l_{2}}\right)^{5}\right)\right], 0 \leq \frac{h}{l_{2}} \leq 1
\end{aligned}
$$

\section{Kết quả và thảo luận}

Để giảm thiểu thời gian tính toán cũng như vẽ đồ thị, nghiên cứu sử dụng phần mềm Mathcad. Do kích thước răng lưỡi cưa nhỏ (bước răng $\mathrm{T}=$ $12 \div 18 \mathrm{~mm}$, chiều cao răng $6 \div 10 \mathrm{~mm})(\mathrm{A} \& \mathrm{~B}$ TOOLS (2021), yêu cầu cơ cấu cam và tay quay con trượt cũng phải phù hợp. Sau khi tham khảo kích thước của một vài máy mài lưỡi cưa vòng xẻ gỗ dẫn động bằng cơ cấu cam, nghiên cứu đã chọn tay quay con trượt có chiều dài thanh truyền bằng $600 \mathrm{~mm}$, tay quay có chiều dài $150 \mathrm{~mm}$, độ dịch chuyển cần tịnh tiến lớn nhất $50 \mathrm{~mm}$ làm thông số đầu vào. Các hàm số xác định được xây dựng theo biến góc quay của tay quay $\varphi_{21}$.

Theo nguyên lý mài lưỡi cưa, điều kiện tiên quyết tránh va đập đá mài vào lưỡi cưa là chuyển động đẩy lưỡi cưa đi, đá mài phải chuyển động xuống. Kết quả pha chuyển động của hai cơ cấu được tính toán bằng Mathcad. Hình 6 cho thấy, hai chuyển động là ngược nhau với tốc độ di chuyển ra của cần đẩy cam khá lớn và nhanh.

Đối với cơ cấu tay quay con trượt, khi các thông số hình học được xác định, có thể xây dựng bản vẽ thiết kế. Tuy nhiên, với cơ cấu cam, biên dạng cam cần phải xác định, nó không chỉ khống chế hành trình mà dạng đường cong bề mặt cam quyết định đến vận tốc, gia tốc cần đẩy. Nhờ vào thiết lập đa thức bậc 5, biên dạng cam được xác định và chỉ trên Hình 7 . Trong hành trình chuyển động tịnh tiến của con trượt từ $0 \div 0,15 \mathrm{~m}$, cần đẩy

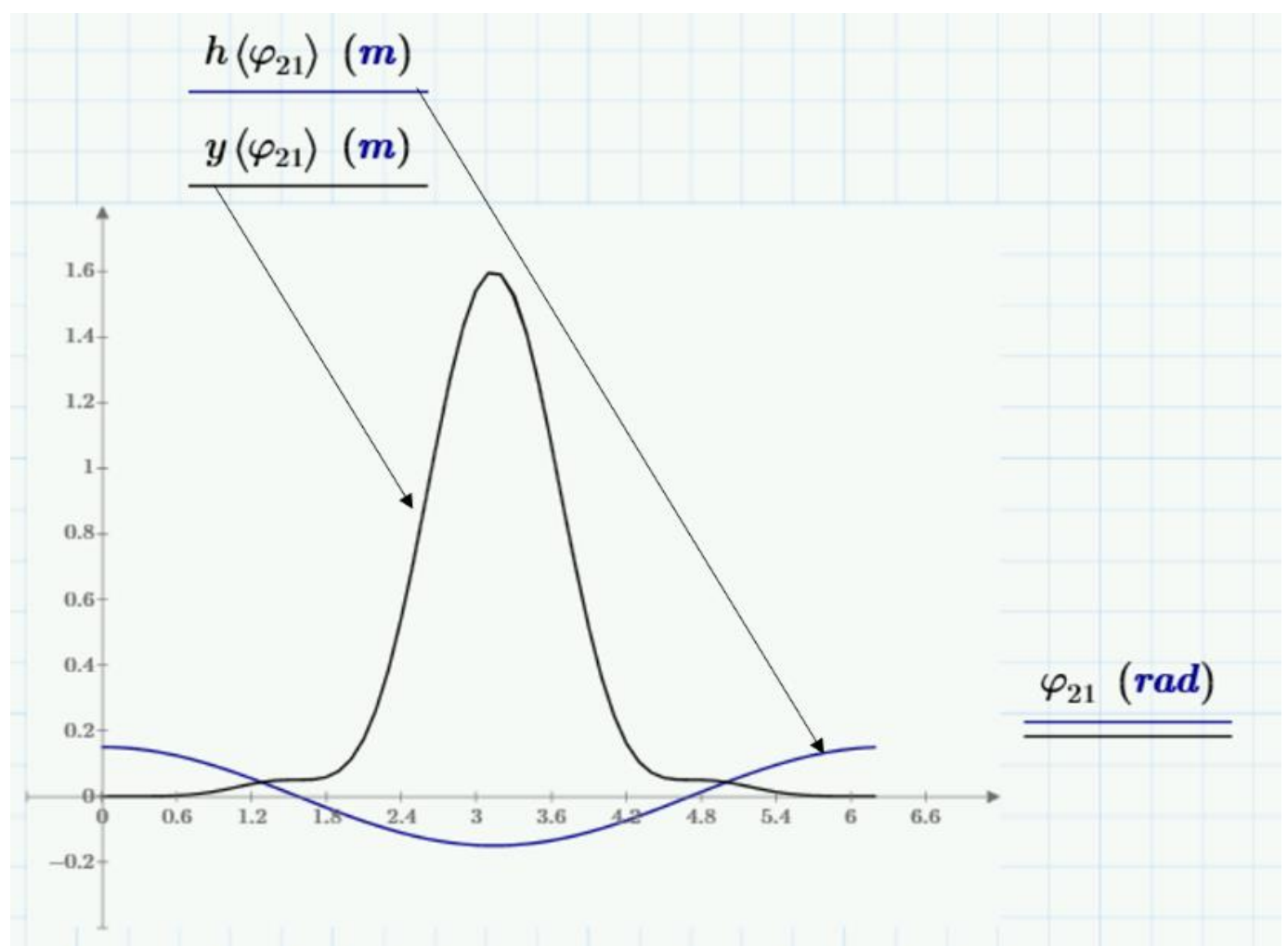

Hình 6. Pha chuyển động của con trượt và cần cam tịnh tiến. 


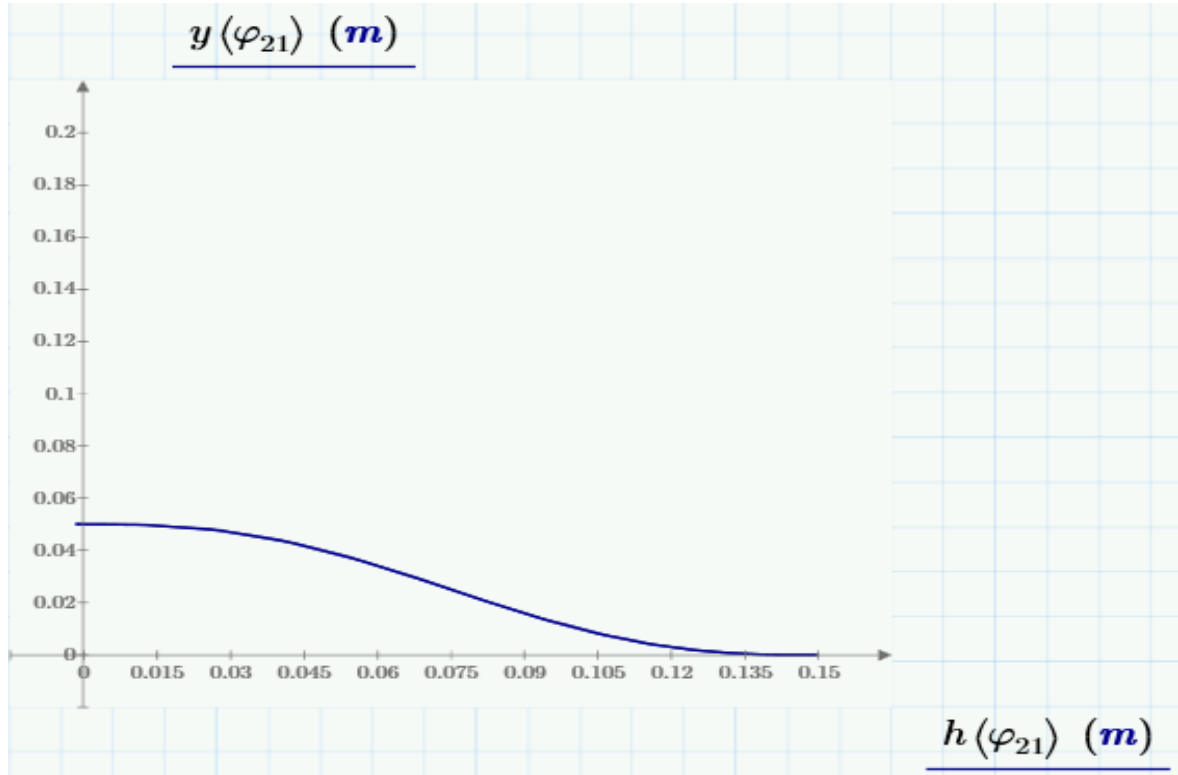

Hình 7. Biên dạng mặt cam.

di chuyển quãng đường tương ứng từ $0,05 \mathrm{~m}$ đến 0 .

Để đảm bảo an toàn, không có va đập cam với cần đẩy, trên đồ thị vận tốc và gia tốc cần đẩy không có bước nhảy. Đạo hàm theo góc quay tay quay để xác định vận tốc, gia tốc cần cam, kết quả tính được trình bày trên Hình 8. Căn cứ đồ thị cho thấy, không có bước nhảy trên đồ thị, do đó biên dạng cam trên Hình 8 đạt yêu cầu.

\section{Kết luận}

Để xác định được các kích thước hình học của cơ cấu tay quay con trượt kết hợp với cơ cấu cam tịnh tiến nhằm dẫn động cho máy mài lưỡi cưa

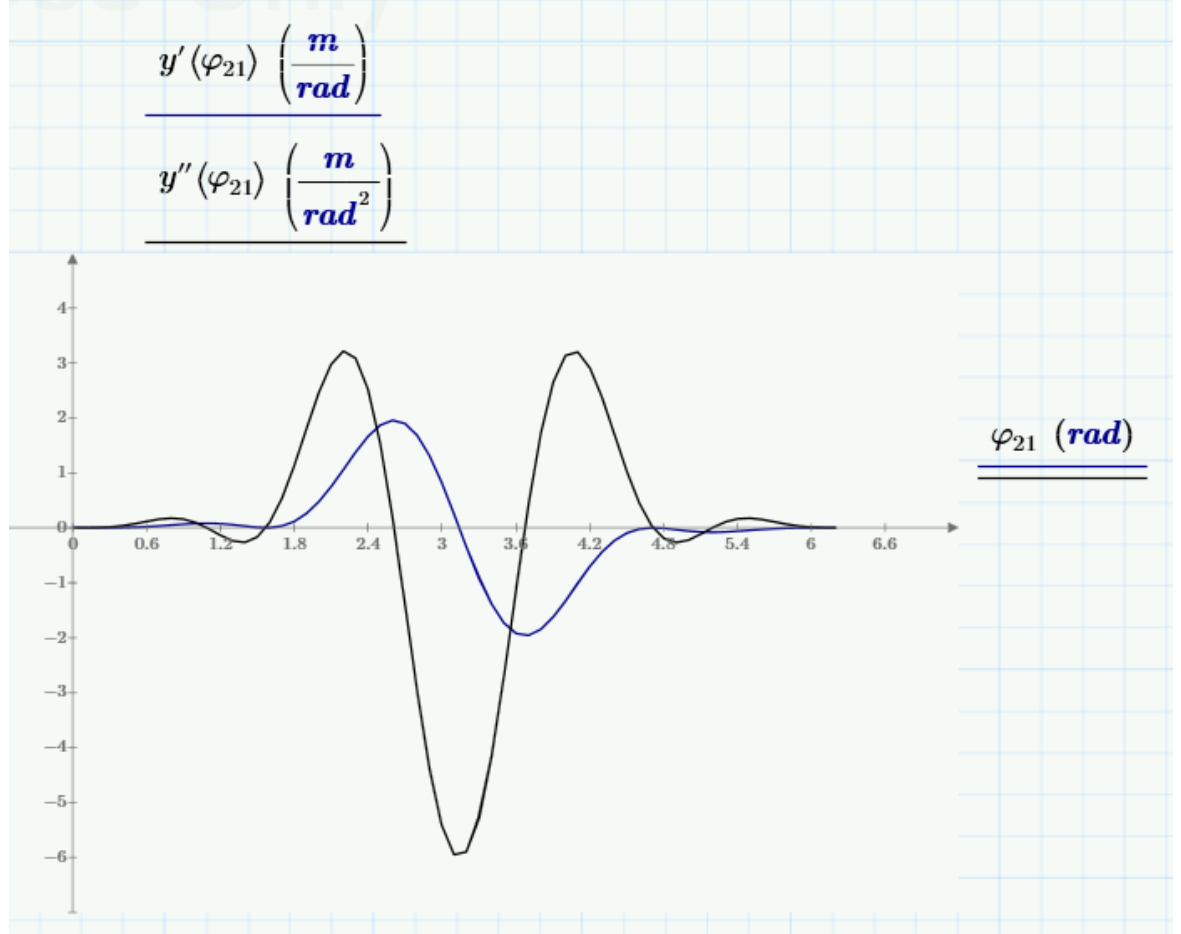

Hình 8. Vận tốc, gia tốc cần đẩy. 
vòng tự động, bài báo này đã lựa chọn phương pháp giải tích. Khi thay đổi các thông số đầu vào của cơ cấu tay quay con trượt như chiều dài thanh truyền, chiều dài tay quay, hành trình cần đẩy cơ cấu cam, phương pháp giải tích với sự hỗ trợ của phần mềm Mathcad cho phép tính và vẽ đồ thị mối quan hệ giữa các pha chuyển động của cơ cấu cam và tay quay con trượt nhanh chóng.

Sự kết hợp giữa cơ cấu cam cần đẩy và cơ cấu tay quay con trượt được ứng dụng trên máy mài lưỡi cưa vòng tự động. Nghiên cứu này có thể mở rộng phạm vi sử dụng cho cơ cấu cam cần lắc sử dụng cho các máy và thiết bị khác nhau như trên các máy dệt, máy đóng gói hay các công đoạn lắp ráp.

\section{Đóng của các tác giả}

Nguyễn Đăng Tấn: tổng hợp bài báo, nghiên cứu xây dựng biên dạng cam, tính toán và vẽ đồ thị các pha chuyển động, biên dạng cam, vận tốc và gia tốc của cần đầy; Lê Thị Hồng Thắng: thu thập tài liệu tham khảo phần tiếng Việt, tìm hiiểu chung về lưỡi cưa vòng, cấu tạo và nguyên lý làm việc của máy mài lưỡi cưa vòng tự động; Đoàn Văn Giáp: xác định các thông số động học của cơ cấu tay quay con trượt theo góc của tay.

\section{Tài liệu tham khảo}

Andreas Fricke, Detlef Günzel, Thomas Schaeffer. (2015). Bewegungstechnik. Carl Hanser Verlag.
Corvers B., Hüsing, M., (2015). Grundlagen, Entwicklung und Anwendung ungleichmäßig übersetzender Getriebe. 5 Auflage, Springer Vieweg.

Đinh Gia Tường, Tạ Khánh Lâm. (2003). Nguyên lý máy. Tập 1, 2 Nhà xuất bản Giáo dục, Hà Nội.

Luck K., Modler K-H.(1990). Getriebetechnik. Springer Verlag Wien.

Maik Berger, (2011). Kolloquium Getriebetechnik Tagungsband. Technische Universität Chemnitz,

Nguyễn Trong Hiệp, (1997). Chi tiết máy. Tập 1, 2. Nhà xuất bản Giáo dục, Hà Nội.

Jack P. Holman, John R. Loyd. (1999). Design of machinery. Second edition, McGraw-Hill Inc

Johannes Volmer. 1989. Kurvengetriebe. VEB Verlag Technik Berlin.

Trịnh Chất, Lê Văn Uyển (2000). Tính toán, thiết kế hệ dẫn động cơ khí. Tập 1, 2, NXB Giáo dục, Hà Nội.

Trần Minh Quang, Nguyễn Quang Hưng. (2015). Thiết kế cơ cấu cam cần đẩy đáy con lăn với sự trợ giúp của máy tính. Trang 125-130, Tạp chí Khoa học và Công nghệ.

A\&B TOOLS (2021).“Lưỡi Cưa Vòng Bản Rộng Cắt Gỗ - Lưỡi Cưa CD “, Internet: http://abtools.com.vn/.

Nhật Phương (2021). "máy mài lưỡi cưa vòng tự động ", Internet: http://nhatphuong.com.vn. 